Stipe Perišić $\bowtie$

Jani Barle

Predrag Đukić

Hinko Wolf

https://doi.org/10.21278/TOF.451026321

ISSN 1333-1124

eISSN 1849-1391

\title{
A BAYESIAN CONJUGATE MODEL FOR THE ESTIMATION OF FRICTION INTENSITY
}

\begin{abstract}
Summary
This paper addresses the Coulomb dry friction force as a technical indicator for fast and efficient condition-based maintenance. To estimate the value of friction force, the Bayesian analysis is used. Instead of the complex Markov Chain Monte Carlo numerical method, a closed-form analytical solution is applied. Thus, a simple and efficient procedure for friction estimation is described. Such a solution in the Bayesian context is known as the conjugate prior. The procedure presented here is verified numerically and experimentally by directly comparing the estimated value with the measured one. Two families of conjugate priors, the gamma-exponential and the normal-gamma, are compared. It is shown that the latter is suitable for friction estimation. An additional parameter, the precision parameter, was proposed as a criterion for the acceptance of estimation.
\end{abstract}

Key words: $\quad$ Bayesian inference, Conjugate priors, Coulomb dry friction, Experimental friction estimation

\section{Introduction}

From the diagnostic perspective, the parameter estimation is a key aspect in a successful damage assessment and consequently in failure prediction. Generally, in diagnostics, if some measurable value is well correlated with the system condition, it can be used as a technical indicator. When considering the cases involving mechanical contact, the friction force is the first choice for an indicator. The problem that arises is how to develop fast and efficient data processing, which can be further included in the condition-based maintenance (CBM) [1-3]. Since mechanical contacts are unwanted occurrences, they should be detected in the early development stage. Therefore, an additional requirement is the ability to detect small quantities. A general CBM approach with two phenomenologically different distributions is presented in Fig. 1. The parameter density distribution, $f\left(r \mid T_{i}\right)$, is related to the system condition at a given time instance. It captures the technical indicator uncertainty due to different sources, for example the sensor noise and ambient condition. As the system deteriorates over time, the indicator value changes. This is observed by diagnostic detection points noted as $T_{1}, T_{2}$ and $T_{n}$ in Fig. 1. Since the failure is a future event, the failure distribution, $f\left(\Delta t \mid T_{n}\right)$, is a model of the remaining useful life. To predict failure, the threshold 
should be preset. Then, with more detection points, a better prediction is obtained [4]. The focus of this paper is not on the failure level settings or the CBM prediction phase but on a technically simple and efficient time-domain procedure for the estimation of friction.

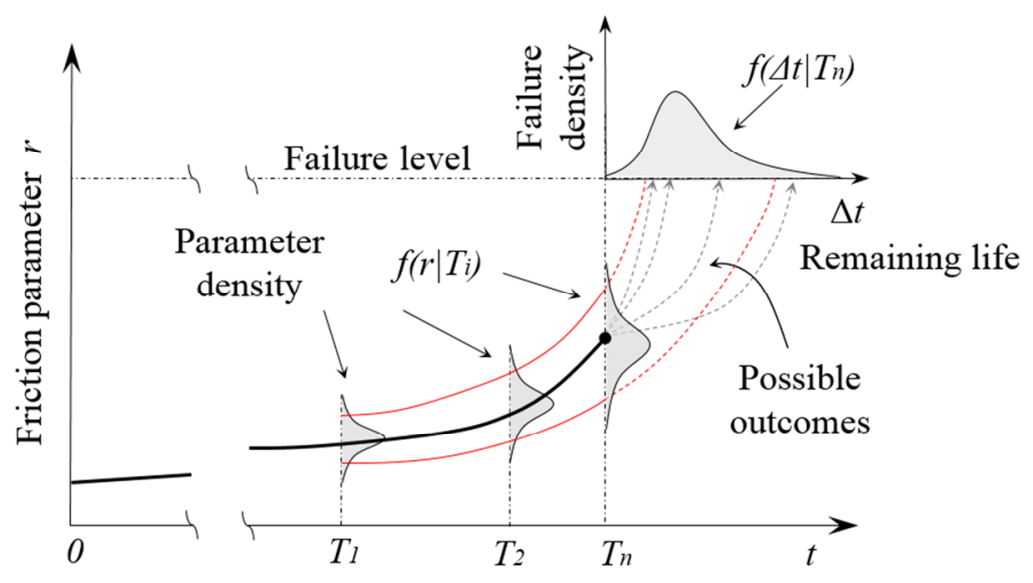

Fig. 1 A schematic diagram of condition-based maintenance

For the systems with a stationary operating point, diagnostics can be performed without detecting its intrinsic values. Subsequently, a simple amplitude analysis, such as RMS, can be performed [4]. The analysis that will be presented here, applies to the systems with a dominant first mode, with frequent changes of the operating point. Therefore, short vibrating intervals without external energy input are expected. Within the conventional identification approach, an extended interval of free decay periods must be acquired. From the diagnostic standpoint, this is not feasible and friction estimation should be performed by analysing just a few periods. Therefore, it is crucial to have an additional criterion to evaluate the estimation before acceptance.

For most mechanical systems, the aging process causes a bigger change in the friction force $(r)$ than in the mass $(m)$ and the stiffness $(k)$, such as oil film loss or lubricant deterioration. In those cases, direct methods and more complex monitoring can be used [5]. This paper presents the way how to use the friction force as a technical indicator directly correlated to damage, but indirectly measured from system response. Herein, the second-order mechanical system with dry friction is analysed. Among friction models [6,7], the Coulomb model is a model which is commonly used due to its simplicity [8-10]. It can be found as a damping model in dry and foil gas bearings [11,12], or in cases involving oil film loss $[13,14]$. When the Coulomb friction model is applicable, the equation of motion is Eq. (1).

$$
m \ddot{x}+r \operatorname{sign}(\dot{x})+k x=f(t)
$$

For the free decay case, the analytical solution exists and can be found in $[15,16]$. It is a well-known fact that in the presence of dry friction, free-response amplitudes decay linearly, i.e. the envelope is a straight line with a constant slope [17]. The slope, and therefore the friction force, can be estimated from at least two consecutive amplitudes. Due to the noise and uncertainty, the friction estimation must be in the form of a distribution. In this paper, any discrepancy difference between the model and the acquired signal, regardless of the cause, will be considered as noise. More details about this can be found in section 4 . In the real conditions, the Bayesian analysis provides the parameter estimation from the first detection instance [18]. It combines the prior distribution with some evidence to obtain a posterior distribution [19]. Since the system changes due to degradation, as shown in Fig. 1, the succeeding runs cannot be taken as a sample from the same population. The parameters are then monitored through an update process. This means that the posterior distribution from the previous detection point becomes the prior distribution to the current detection point. To 
estimate the parameter value, as many detection instances as possible should be considered as successful. The Bayesian analysis presented here will provide a means for accepting and estimating the parameter, even when a small data set occurs. Due to multidimensionality, the Bayesian analysis usually implies the Markov Chain Monte Carlo (MCMC) numerical integration. This is an efficient but complex method that involves an additional sampling algorithm [20,21]. A different parameter estimation approach can be used instead by applying an analytical solution, i.e. a conjugate prior. From an engineering standpoint, these analytical solutions should be considered.

Therefore, a fast and efficient friction estimation procedure using a conjugate prior is proposed in this paper. In brief, the conjugate prior is the case when the prior and the posterior distribution have the same algebraic form [22]. There are a few conjugate priors and they are mainly applicable to one-dimensional problems. Here, to estimate the Coulomb friction force, two conjugate models are compared, namely a simple one-dimensional gamma-exponential model [23] and a two-dimensional normal-gamma model [24]. Also, uncertainty is relatively easily estimated within the same theoretical assumption by calculating the credibility intervals, often on a very small data set. In the CBM diagnostic phase, due to the small data set, the credibility intervals are expected to be wide. Therefore, an additional precision parameter is proposed as a criterion for the acceptance of signal and estimation.

To demonstrate the proposed procedure in a real, but controlled environment, a single degree of freedom testbed is developed. The main feature of the testbed is precise vertical motion with minimal damping, which is achieved through an air bearing [25]. The friction force is induced externally and estimated on the acceleration free decay signal. The estimated friction force distribution is compared to the friction force directly measured by a force sensor.

\section{The Bayesian conjugate prior for friction estimation}

According to Eq. (1), the amplitudes in the free response decays linearly. The friction force, $r$, and the envelope free decay slope, $y$, are related by Eq. (2), where $\omega_{0}$ is the natural frequency, while $k$ is the stiffness [17,26].

$$
y=\frac{2 r \omega_{0}}{\pi k}
$$

A series of ideal measurements will yield the same slope for any consecutive two peaks. In the real-life signal, this is not the case and different values are estimated even for different amplitudes of the same run. Since uncertainty is present, a Bayesian analysis is a reasonable choice for parameter density [20], i.e. friction force estimation, at any detection point, as shown in Fig. 1. The parameter density consists of the aleatory and the epistemic uncertainty $[27,28]$. The former is caused by the loss of signal information, such as the noise which or an improper sampling rate. The epistemic uncertainty is due to the selection of a model. The Bayesian mathematical expression is shown by Eq. (3).

$$
\pi_{1}(\boldsymbol{\varphi} \mid x)=\frac{f(x \mid \boldsymbol{\varphi}) \pi_{0}(\boldsymbol{\varphi})}{\int f(x \mid \boldsymbol{\varphi}) \pi_{0}(\boldsymbol{\varphi}) d \boldsymbol{\varphi}}
$$

This expression is a combination of the prior distribution, $\pi_{0}(\varphi)$, of the parameter vector, $\boldsymbol{\varphi}$, and the likelihood function, $f(x \mid \boldsymbol{\varphi})$, which yield the posterior distribution, $\pi_{1}(\varphi \mid x)$. The prior distribution represents the belief about the parameter value before the data has been acquired. The choice of the prior distribution must be supported by an expert opinion or previous knowledge to ensure the convergence. A too wide or narrow prior distribution means very slow convergence [23]. The likelihood function is a statistical model that defines 
the difference between the acquired data and the proposed model [29]. The prior distribution and the likelihood function form the numerator, which is scaled by a denominator, often referred to as the normalization constant.

The Bayesian updating process is as follows. When the first data set (free decay), which corresponds to the detection point $T_{1}$ in Fig. 1, is acquired and adopted, the likelihood function and the prior distribution form the first posterior distribution. After the second data set, the detection point $T_{2}$, the posterior distribution from $T_{1}$, should be taken as the prior distribution for $T_{2}$, and so on. Thus, in the updating process, different data sets are used to establish the density $f\left(r \mid T_{i}\right)$. In the updating circle from the prior to the posterior distribution, there are some combinations which yield a fully analytical, closed-form solution for Eq. (3). This solution is referred to as a conjugate prior. The conjugate prior implies the prior and the posterior distribution in the same functional form leading to a highly efficient parameter estimation. The list of conjugate priors is limited, and the selected pair must be well-argued and justified by an example. In this paper, two families of conjugate priors will be addressed.

In every real-life system response noise is present and it is typically modelled according to a normal distribution. Therefore, it would be reasonable to assume the same random model for the slope derived from every successive amplitude. Based on this assumption, the estimation of friction can be performed via a conjugate prior. As stated previously, there is a limited number of conjugate priors, but when the model is a normal distribution, the normalgamma distribution presented here in Eq. (4) is a conjugate prior.

$$
\pi_{1}^{G N}\left(r, s \mid \mu_{H}, \lambda_{H}, \beta_{H}, \alpha_{H}\right)=\frac{\beta_{H}^{\alpha_{H}} \sqrt{\lambda_{H}}}{\Gamma\left(\alpha_{H}\right) \sqrt{2 \pi}} s^{\alpha_{H}-\frac{1}{2}} e^{-\beta_{H} s} e^{-\frac{\lambda_{H} s}{2}\left(r-\mu_{H}\right)^{2}}
$$

This is a bivariate distribution, and $r$ and $s$ in Eq. (4) are the variables, i.e. the friction force and the precision parameter, respectively, which are the system parameters of interest. The precision parameter is just the inverse of variance, so this family of conjugate priors provides an estimation of the average deviation between the proposed model and the acquired data. However, to obtain only the friction force distribution, as shown in Fig. 1, marginalization has to be performed. Since the complex friction process is approximated by the Coulomb model, the friction force parameter variance can be large. In that case, the precision parameter is chosen as a control parameter to additionally support the estimation. The notations $\mu_{H}, \lambda_{H}, \beta_{H}, \alpha_{H}$ represent the distribution parameters. The distribution parameter $\mu_{H}$ is related to the mean value of $r$, with the standard deviation related to $\lambda_{H}$. The parameters $\alpha_{H}$ and $\beta_{H}$ refer to the precision parameter $s$. The mean value and the standard deviation are set according to the principles of gamma distribution. This means that the mean value is the ratio between $\alpha_{H}$ and $\beta_{H}$ with a standard deviation of $\alpha_{H} / \beta^{2}{ }_{H}$. Equation (4) represents the prior and the posterior distribution. The only difference between them is in the parameter values. Parameters in Eqs. (5-8) marked with the subindex 0 are related to the prior distribution which reflects our initial about the system parameters $r$ and $s$. Regardless of the acquired data, these parameters are set according to the initial information (expert opinion). The parameters marked with $H$ are the so-called hyper-parameters related to the posterior distribution. They are a result of derivation from Eq. (3) and can be calculated according to Eqs. (5- 8)

$$
\begin{aligned}
& \mu_{H}=\frac{\lambda_{0} \mu_{0}+n \bar{r}}{\lambda_{0}+n} \\
& \lambda_{H}=\lambda_{0}+n \\
& \alpha_{H}=\alpha_{0}+\frac{n}{2}
\end{aligned}
$$




$$
\beta_{H}=\beta_{0}+\frac{1}{2} \sum_{i=1}^{n}\left(r_{i}-\bar{r}\right)^{2}+\frac{n \lambda_{0}}{\lambda_{0}+n}{\frac{\left(\bar{r}-\mu_{0}\right)^{2}}{2}}^{2}
$$

In Eqs. (5-8), $\bar{r}$ is the mean of samples, while $n$ is the number of considered data. Additional information on this conjugate prior model can be found in [24] and similar information on the chi-square conjugate prior model in [30].

The objective of Bayesian analysis is to use the simplest possible model. Therefore, the previous model will be compared to the frequently used one-dimensional gamma-exponential model [23], represented by Eq. (9).

$$
\pi_{1}^{G E}\left(r \mid \kappa_{H}, \alpha_{H}\right)=\frac{\kappa_{H}^{\alpha_{H}}}{\Gamma\left(\alpha_{H}\right)} r^{\alpha_{H}-1} e^{\left(-\kappa_{H} r\right)}
$$

Notations $\alpha_{H}$ and $\kappa_{H}$ represent hyper-parameters, which can be calculated by Eqs. (1011). The subindex 0 denotes prior parameters, while $r$ and $n$ are the friction forces and the number of considered data, respectively.

$$
\begin{aligned}
& \alpha_{H}=\alpha_{0}+n \\
& \kappa_{H}=\kappa_{0}+\sum_{i=1}^{n} r_{i}
\end{aligned}
$$

As it will be shown, this is a rather fast and efficient procedure, and as long as one parameter is to be estimated, the proposed method applies to any system. Otherwise, the more complex MCMC method has to be used.

\section{Numerical example of friction estimation}

To demonstrate the efficiency of the friction estimation procedure, numerically simulated data with known parameter values were analysed. By doing so, the epistemic uncertainty, which is always present when dealing with a real-life problem, is completely excluded from the analysis. The Coulomb friction force is set to $0.07 \mathrm{~N}$, the mass is $10 \mathrm{~kg}$ and the stiffness is $1000 \mathrm{~N} / \mathrm{m}$. The Gaussian noise with a zero mean value and a standard deviation $(\sigma)$ of $5 \mathrm{e}-4$ were added to the simulated response. This yields a signal-to-noise ratio (SNR) of $20 \mathrm{~dB}$.

The friction force is assumed to be scattered around the true mean according to a normal distribution. To test the assumption initially, the same data were analysed with the normal distribution probability paper. It was found that data can be well approximated with a straightline, which preliminary proves the model. In this example, the total length of the analysed signal was 33 seconds, and all of the 51 amplitudes (evidence) were included in the Bayesian analysis according to Eqs. (5-8). The graphical representation of the simulated signal is omitted due to brevity.

The estimated parameters are shown in Fig. 2. The upper panel represents the prior distribution, while the lower one represents the posterior distribution after all data (amplitudes) have been considered. It is important to mention again that the noise model added to the smooth numerical waveform was a normal distribution with a zero mean and a standard deviation $(\sigma)$ of 5e-4. Since only peak amplitudes were analysed, the waveform noise surely differs from the one related to the amplitudes. This is an important fact since, here, the precision parameter is directly related to the standard deviation. Hence, the true value of this parameter is unknown. This can be considered as a disadvantage of the procedure. Nevertheless, the standard deviation can be roughly estimated via the root mean square error (RMSE), and its value is $4.3 \mathrm{e}-4$. This provides a reference value by which the Bayesian analysis should converge. 
A minor deviation of $0.8 \%$ exists between the true and the estimated friction force, which is $0.0706 \mathrm{~N}$. The estimated value of precision parameter is 34.3 , converting to the standard deviation, whose value is $8.4 \mathrm{e}-4$, which is the same order of magnitude as RMSE. Furthermore, the RMSE estimation falls within the $90 \%$ posterior credibility interval. This is found to be acceptable, particularly considering the prior setup. Generally, the prior parameters are chosen with sparse knowledge about the true value of each parameter, and the expected value differs from the true one. Therefore, the prior distribution should cover the value that it converges to. Due to different limits of axes, which are not immediately visible, the posterior distribution significantly reduces intervals. The $90 \%$ credibility interval now covers a much smaller area compared to the prior distribution. This is particularly noticeable in the case of the precision parameter, which suggests a proper model selection and a good friction parameter estimation.

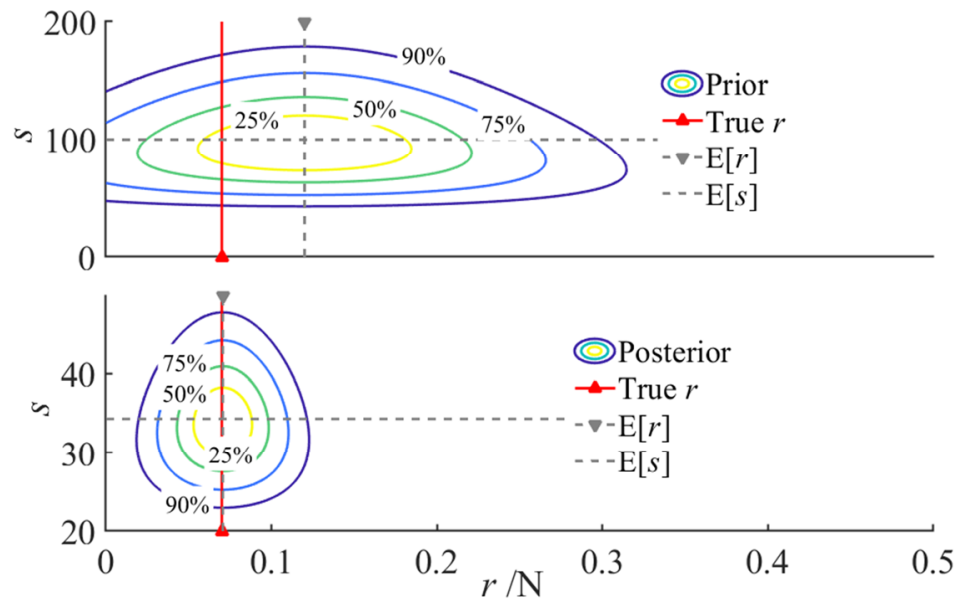

Fig. 2 The Coulomb friction force estimated for a simulated response via the normal-gamma conjugate prior

The impact of aleatory uncertainty is tested on the numerical model, Eq. (1), by increasing the noise levels i.e. $\mathrm{SNR}=14.7 \mathrm{~dB}$ and $\mathrm{SNR}=8.6 \mathrm{~dB}$. When compared to the true friction value, the error is $0.33 \%$ and $2.70 \%$, respectively. Even when the noise level increases, the presented method still remains effective.

Even though the list of conjugate priors is short, the choice is not simple because not every conjugate model is suitable for solving the problem. Thus, on the same data set, the gamma-exponential model, presented by Eq. (9), is analysed. Due to one-dimensionality, this model does not have a control parameter. The prior and the posterior distribution can be seen in Fig. 3 on the top and the bottom panel, respectively. The true value is noted with a vertical red line, while the estimated one with a vertical dashed grey line.

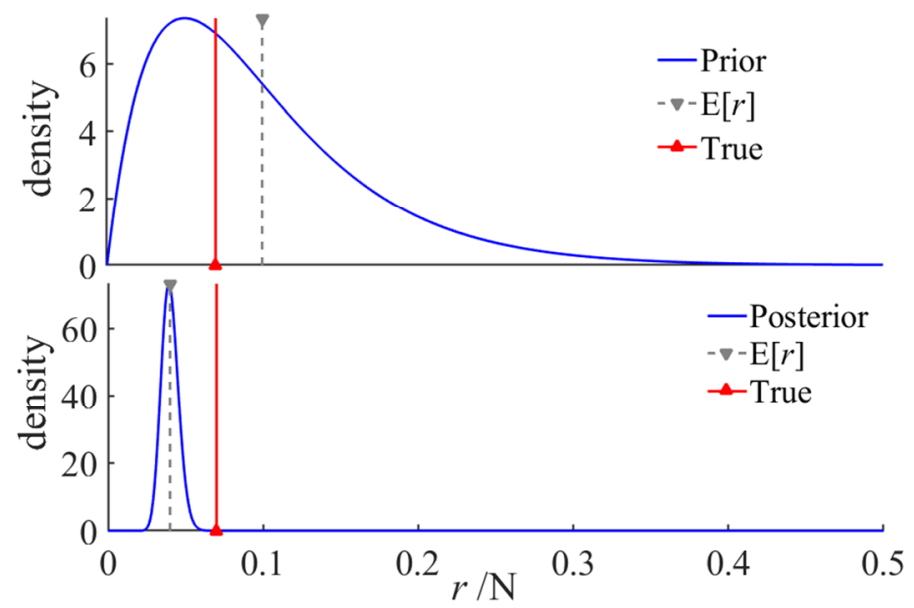

Fig. 3 The Coulomb friction estimated for a simulated response via the gamma-exponential conjugate prior 
Only by observing the posterior distribution width, when the true friction force value is unknown, the result would be accepted for CBM. This is clearly not the case as it converges towards an incorrect value. Since the credibility interval is significantly narrowed and does not include the true value, this conjugate prior is not suitable for the estimation of friction and cannot be applied in a manner shown in Fig. 1; therefore, it will be left out from the further analysis.

The example given in the previous paragraph proves the need to introduce the precision parameter, which can support the decision on estimation acceptance. This can be additionally illustrated by the following example in which the total number of periods included in the Bayesian analysis was varied. Obviously, with more evidence at disposal, the estimation will be better. As shown in Fig. 4, the precision parameter value is correlated to the friction estimation, i.e. a higher value of precision parameter indicates a better friction estimation. Thus, the precision parameter can be a criterion for the diagnostic signal acceptance.

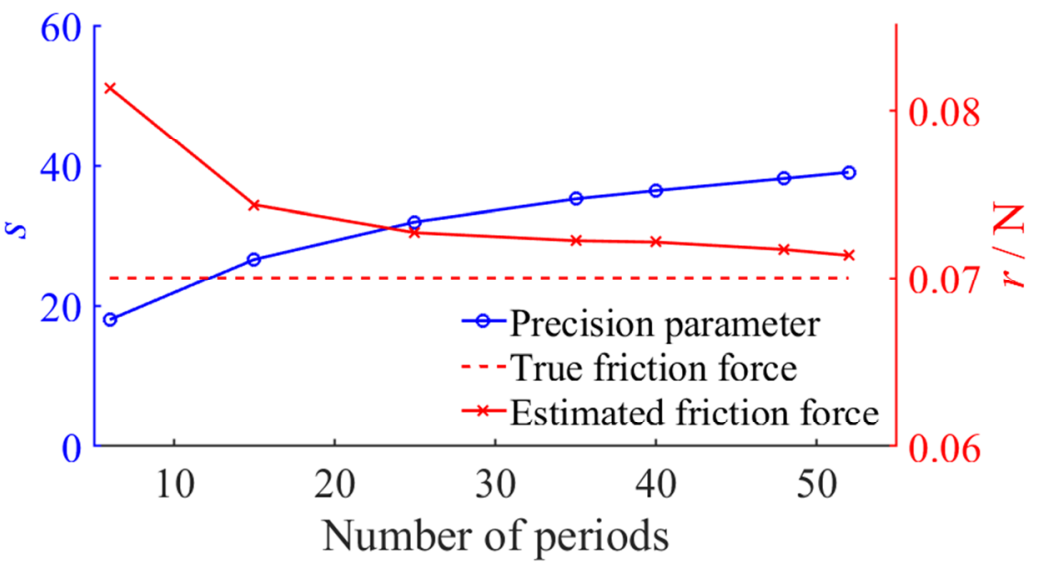

Fig. 4 Precision parameter and the Coulomb friction in correlation with the data used

It should be noted that by considering only the absolute value of precision an erroneous conclusion could be reached. From the diagnostic aspect, it is advisable to have a previously determined reference value and to draw conclusions based on the comparison. More details will be presented in the next section. This numerical example undoubtedly shows that it is possible to obtain an extremely good parameter estimation via the normal-gamma conjugate prior.

\section{Experimental analysis and discussion}

In this section, a testbed shown in Fig. 5 is used to experimentally verify the proposed procedure. Precise, vertical one-dimensional motion is achieved through an air bearing [25]. Since this configuration produces extremely small damping, an external source, i.e. mechanical contact, is added. Friction was induced by the metal contact between the scratching pin on the moving part and the aluminium plate fixed on the stationary part. The force was managed by increasing or decreasing pressure between those two parts. The generated force, which was the value of interest, was directly captured by a force sensor. The system response, which is taken to be a diagnostic signal, was captured by an accelerometer.

All signals were acquired via a NI-USB-6251 National Instrument DAQ device, with a 16-bit resolution and a $1000 \mathrm{~Hz}$ sampling rate. This is more than satisfactory considering the natural frequency of the testbed is $2.9 \mathrm{~Hz}$. No additional filtering of the DAQ system was used. After, the friction force was processed using a Savitzky-Golay filter to refine the reference value. 


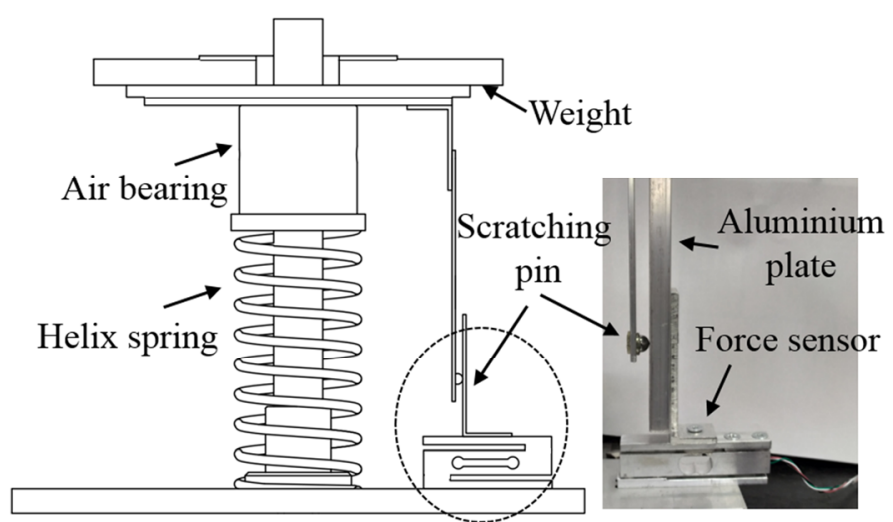

Fig. 5 Experimental setup for the estimation of friction force

As shown in section 3, the estimation of friction will be obtained by fitting the envelope, so correct amplitudes ratios are required for the analysis. The linearity and crosssensitivity of the sensors were verified in detail, before and after the assembly. For acceleration, an ADXL322J sensor was used; its operating range is $\pm 2 \mathrm{~g}$, the noise in this configuration is $6.2 \mathrm{e}-3 \mathrm{~g}$. Calibration was performed using a B\&K accelerometer, Model 4370 , and a B\&K calibrator, Model 4291. The total phase error of the accelerometer signal chain is 0.35 deg. During the operation, an additional displacement sensor was used to check the signal adopted for the analysis. The sensor is Honeywell SPS-L075-HALS with an operating range of $0-225 \mathrm{~mm}$ and linearity $\pm 0.4 \%$. For friction force, a double beam load cell was used, with an operating range of $\pm 1 \mathrm{~N}$. The load cell was recalibrated, and its linearity and crosssensitivity were tested with a set of laboratory weights; it was also back-to-back tested with a Tedea Huntley model 1015 load cell in the overload range.

A typical friction force signal in comparison to the ideal Coulomb model is shown in Fig. 6. Certain differences can be noticed in the whole segment, which is an aleatory uncertainty. But at about 3.8, 4, and 4.5 seconds, the difference is more significant, which is essentially an epistemic uncertainty, i.e. the indication of a more complex model. Since the signal approximation is satisfying, the Coulomb model is adopted. As the system deteriorates, the friction force increases, and a more complex model is taken to be unnecessary because the friction values will be compared relatively. This diagnostic context of the proposed procedure is an additional reason for adopting this model.

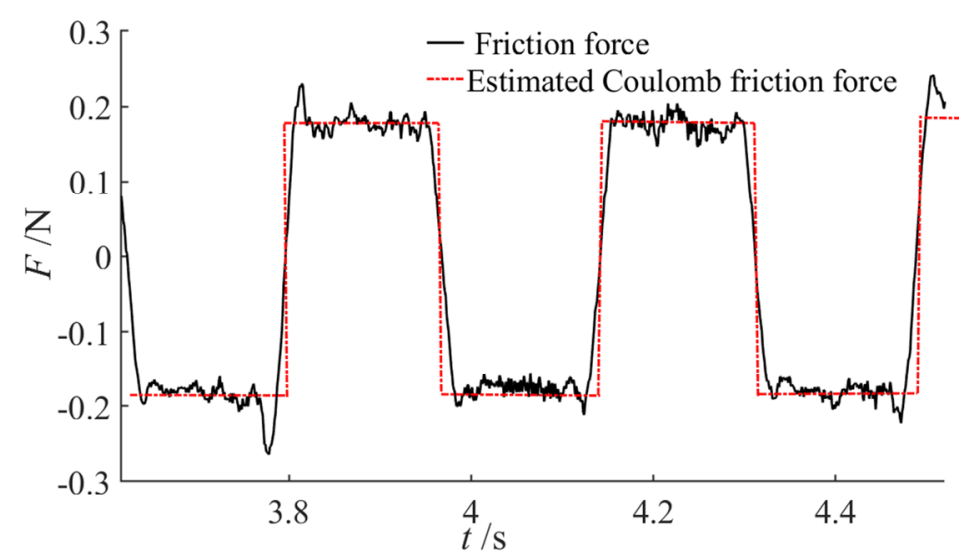

Fig. 6 A typical friction force signal and the Coulomb model adopted

The first example presented here is with a higher value of friction force, i.e., the reference value is $0.2933 \mathrm{~N}$. The acquired acceleration response can be seen in Fig. 7 . 


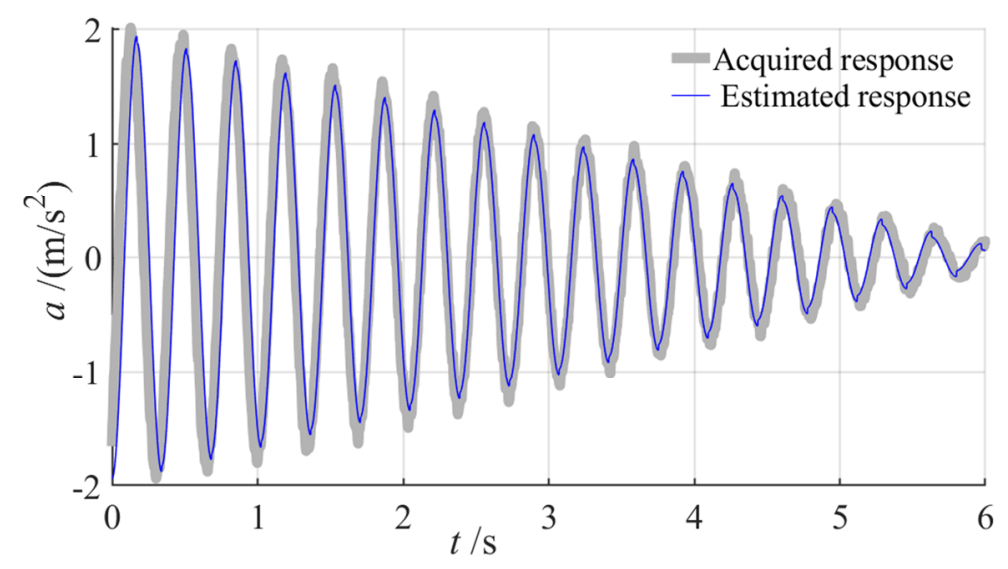

Fig. 7 Decaying acceleration amplitudes for the case with a higher value of friction force

The prior and posterior distributions are shown in Fig. 8. The prior distribution is relatively wide and is set quite far from the reference value, which emphasizes the uncertainty in initial parameter values. Despite the prior distribution mean, its width ensures that the posterior distribution converges near the reference value. The estimate parameters are: a friction force of $0.2909 \mathrm{~N}$ and a precision parameter of 5.7. The estimated values in comparison to reference values can be seen in Table 2 .

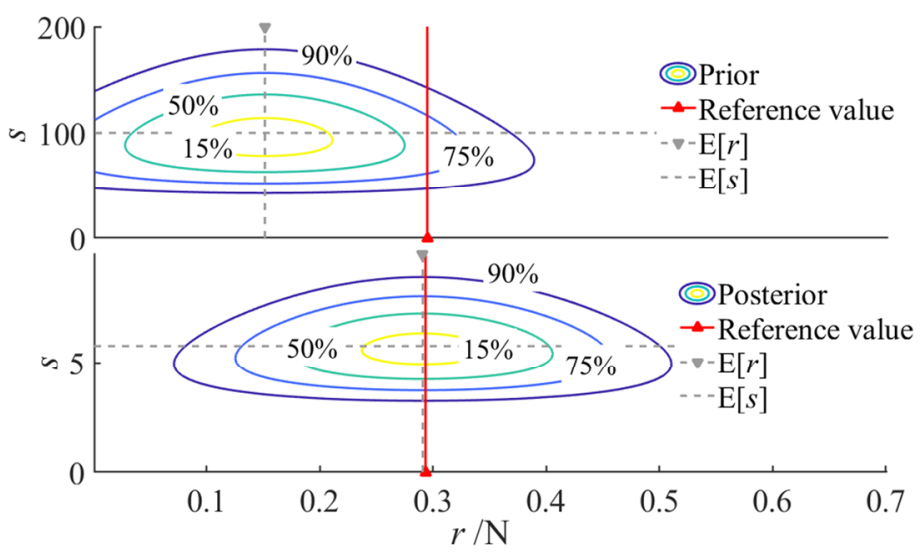

Fig. 8 The Coulomb friction force estimated for the case with a higher value of friction force.

Besides the point estimation, the distributions also offers additional information. As noted earlier, the precision parameter is taken to be a control parameter that evaluates the estimation. As for the precision parameter width, it suggests a proper model selection and a good friction estimation. However, parameters within the selected model differ. This can be seen in Fig. 6, where the acquired friction force varies around the mean considerably. Therefore, the distribution width for the friction force is relatively wide. In these situations, a different prior selection could provide a more certain estimation if needed. Nevertheless, regarding the diagnostic context, the estimation is satisfactory. For example, if this estimation corresponds to the detection point $T_{1}$, as shown in Fig. 1, then the reference value "slides" to the right as the degradation progresses. Then, the relatively wide posterior distribution from the first detection ensures proper updating for the following posterior distribution which corresponds to the point $T_{2}$.

A comparison between the acquired (thick grey) and the estimated (blue) system response is shown in Fig. 7. It is evident that the amplitude decays linearly, and that the relative amplitude ratio is appropriate, which means that the estimation is satisfactory. Also, there is a minor difference between the waveform amplitudes and the periods. This is due to the fact that only one parameter was analysed. Since other system parameters, mass and 
stiffness, also affect the resulting waveform, a small deviation can be attributed to them as well. The characteristic discontinuity due to the friction force is noted on the estimated waveform. This feature is hardly observed in the acquired response due to the sensor noise.

The amplitude decay for the second case analysed here is shown in Fig. 9. The thick grey line represents the acquired response. The reference friction force value is $0.1834 \mathrm{~N}$.

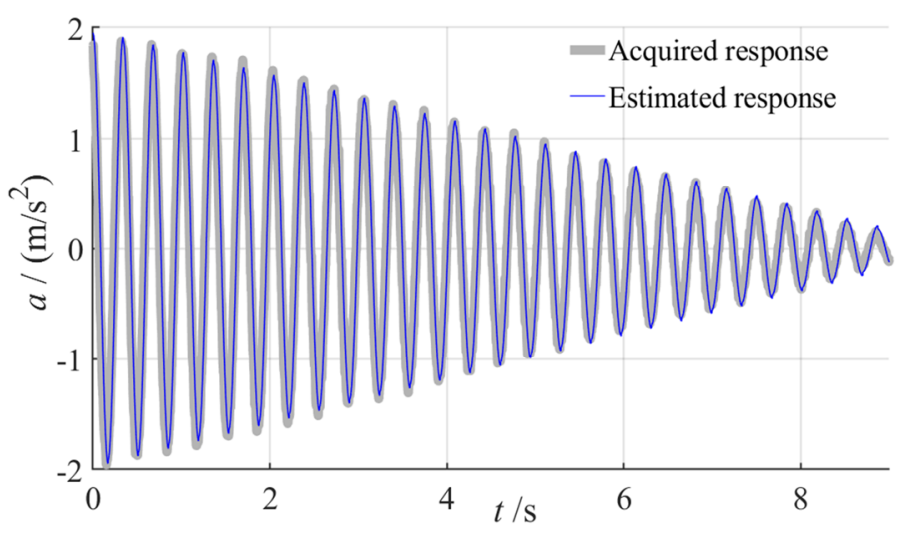

Fig. 9 Decaying acceleration amplitudes for the case with a lower value of friction force

The prior and the posterior distribution can be seen in Fig. 10. Same as in the previous case, the prior distribution is wide and relatively far from the reference value. Here, regardless of the prior setup, good convergence is also noted, and the posterior distribution width is significantly reduced. This suggests lower uncertainty in the parameter estimation. The estimated parameters are the friction force $(r=0.1808 \mathrm{~N})$ and the precision parameter $(s=11.6)$.

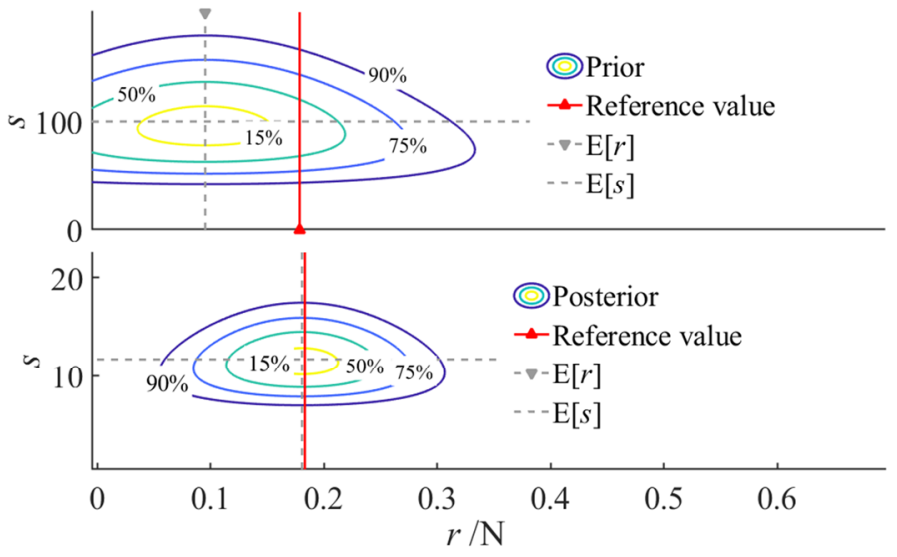

Fig. 10 The Coulomb friction force estimated for the case with a lower value of friction force

The difference between the estimated and the recorded value is around $1 \%$, as noted in Table 1 . However, the precision parameter has a higher value than in the previous case. This implies a smaller deviation between the presumed model and the acquired data, which in turn implies higher confidence in the model. This is caused by a lower value of friction force that affects the total number of periods. If more evidence is used, the confidence is greater. The acquired system response (thick grey line) and the estimated one (blue line) are shown in Fig. 9. Here, the acceleration discontinuity in the estimated waveform is not emphasized as in the previous case, due to a lower value of friction force. The same effect is not observed in the recorded response because of the sensor noise. As already shown, a reasonably good Coulomb friction force estimation can be obtained by analysing the long, free decay responses. In the context of CBM, they do not occur frequently. But, before the diagnostic process began, in the set-up phase, long signals should be used to determine the values of natural frequency $\omega_{0}$ and 
stiffness $k$ for Eq. (2). These parameters, as assumed here, are correlated to degradation to a lesser degree than the friction force $r$ and will not change significantly throughout the process.

A real diagnostic signal with just a few low-intensity periods and with the initial condition unknown is simulated in the next case. Therefore, a short segment at the very end of the signal shown in Fig. 9 is selected. The case to be analysed is shown enlarged in Fig. 15. The total number of periods is more than five times smaller than the full data set. The prior distribution is the same as that for the full data set.

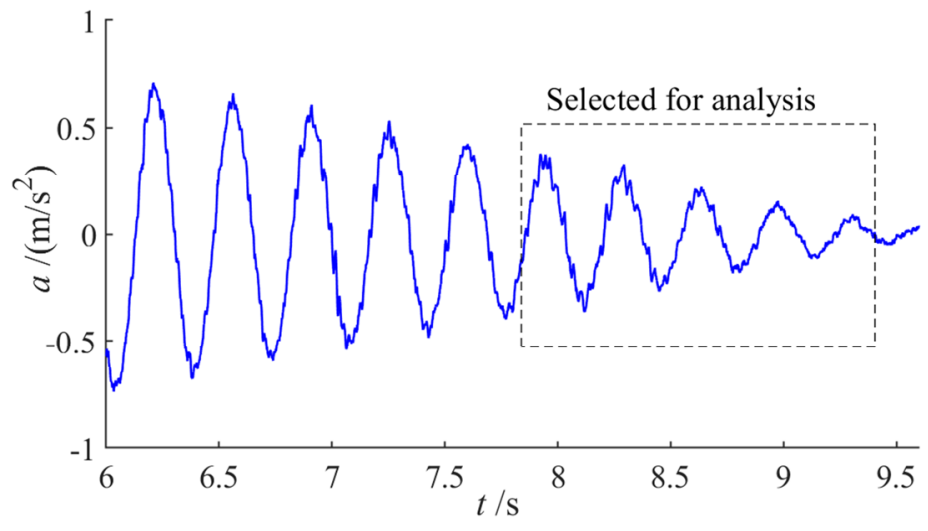

Fig. 11 The acquired signal and a reduced data set

The estimated parameters are shown in Fig. 12. The posterior distribution width in this case is significantly greater, which reflects higher uncertainty. Considering the small data set and the signal-to-noise ratio, it can be concluded that the estimation is reasonably good. The friction force is $0.1643 \mathrm{~N}$, which gives the difference of $10.3 \%$ relative to the reference value, and the precision parameter is 32.2 .

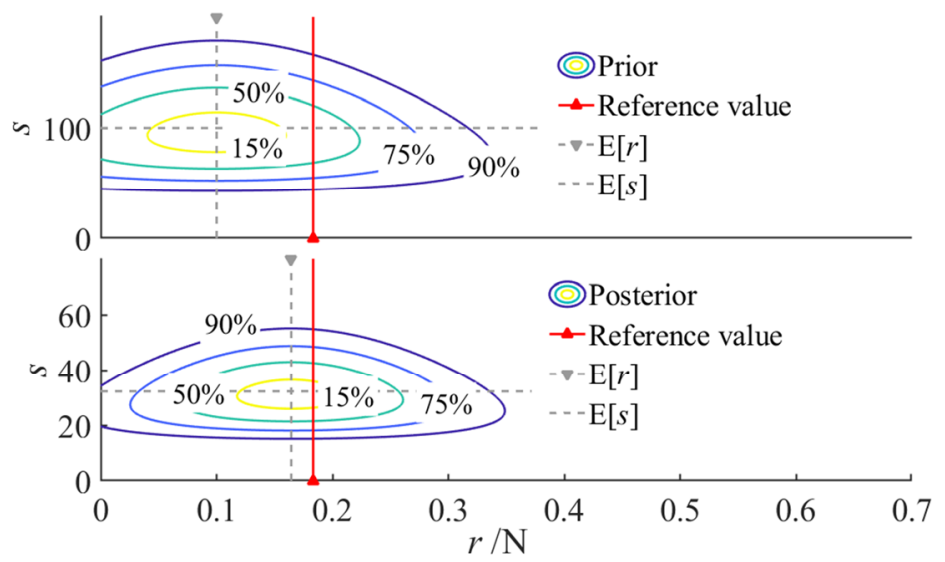

Fig. 12 The Coulomb friction force estimated for the reduced data set

As for the precision parameter, the conclusion would be a more certain estimation compared to the full data set. However, the precision parameter should not be taken as an absolute value, but as a relative one with respect to the value derived in similar conditions. This will be demonstrated later in this section. Here, the high value can be attributed to the prior set up and the lack of data. Nevertheless, the fact that the precision parameter decreases, and the interval is much narrower suggests a good estimation. On the other hand, if we consider only the friction force, the estimation would be rejected because of the large uncertainty involved. A better estimation, if needed, would be achieved with a more suitable prior distribution, such as the one in which the data from the previous detection points are considered. Table 1 shows the estimated friction values in comparison to the reference value for all three cases. 
Table 1 The friction force estimated for all analysed cases

\begin{tabular}{|ll|l|l|l|}
\hline & & Data set $_{1}$ & Data set $_{2}$ & Data set $_{2 \text {-reduced }}$ \\
\hline Estimated value & $r / \mathrm{N}$ & 0.2909 & 0.1808 & 0.1643 \\
\hline Reference value & $r / \mathrm{N}$ & 0.2933 & 0.1834 & 0.1834 \\
\hline Error & $/ \%$ & 0.8 & 1.4 & 10.4 \\
\hline
\end{tabular}

It has been shown previously that the proposed procedure can be efficiently applied in the diagnostic context, i.e. a small number of periods with low intensity. In the following example we consider the quality of the data, which will be reflected in the precision parameter. All the cases presented above are with a $1 \mathrm{kHz}$ sampling rate. To simulate $100 \mathrm{~Hz}$ and $10 \mathrm{~Hz}$ sampling rates, the down-sampling of the signal in Fig. 11 is performed and shown in Fig. 13. Thus, by reducing the sampling rate, some pieces of information are lost. In other words, the aleatory uncertainty increases while the epistemic uncertainty remains the same as the previous one.

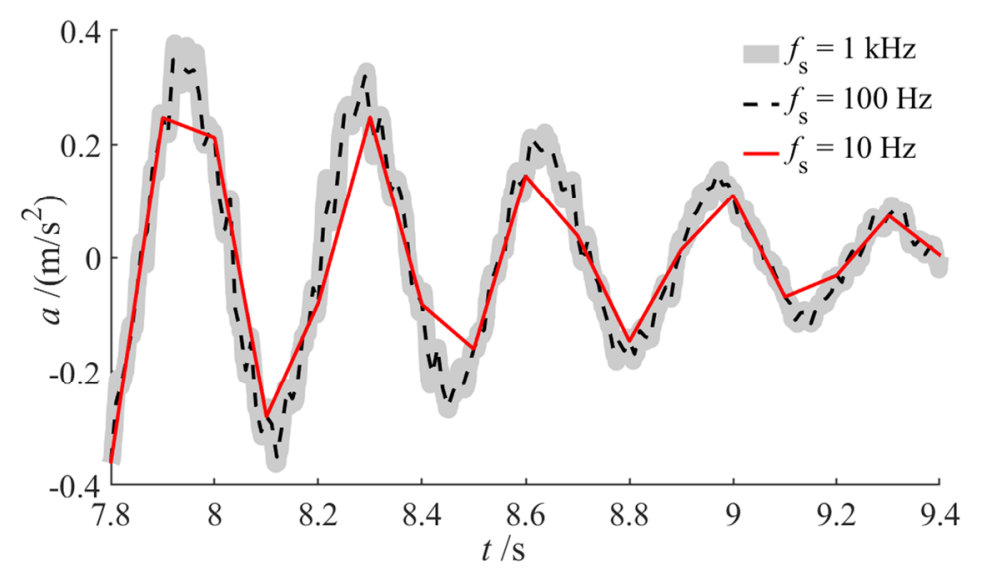

Fig. 13 A reduced data set with different sampling rates

The prior distribution for all three sampling rates is shown in the top panel of Fig. 12, while the posterior distributions are shown in Fig. 14. It is apparent that the discrepancy between the reference and the estimated friction force increases as the sampling frequency decreases, which can be seen in Table 2. The first column in Table 2 corresponds to the third column in Table 1, but here, the precision parameter value is added.

Table 2 The friction force estimated for the reduced data set and different sampling rates

\begin{tabular}{|ll|l|l|l|}
\hline Sampling rate & $f_{s} / \mathrm{Hz}$ & 1000 & 100 & 10 \\
\hline Reference value & $r / \mathrm{N}$ & 0.1834 & 0.1834 & 0.1834 \\
\hline Estimated value & $r / \mathrm{N}$ & 0.1643 & 0.1567 & 0.1465 \\
\hline Error & $/ \%$ & 10.4 & 14.4 & 20.1 \\
\hline Precision parameter & $s$ & 32.2 & 32.9 & 27.5 \\
\hline
\end{tabular}

The precision parameter $s$ is used to propose the estimation acceptance criteria. In this experiment, the value of interest is the friction force $r$; it was directly measured, and the error could be defined, which is not always possible in real life.

The signal sampled with a $100 \mathrm{~Hz}$ sampling rate still represents the system response well, and there is no significant information loss. A small discrepancy between the estimated value of friction and a $1000 \mathrm{~Hz}$ sampling rate exists, but the precision parameter is practically the same. When the sampling frequency is further reduced to $10 \mathrm{~Hz}$, information loss occurs, the error is twice as large and negatively correlated to the precision parameter, as shown in Table 2. 
Although all three signals are free decay signals, the precision parameters manage to detect the signal quality and distinguish the better from the worse. Thus, this parameter can be used as an acceptance criterion when the value of interest cannot be directly measured. For this particular case, the acceptance criterion could be set to 30 . This is something that should be predefined during the setup phase on a sufficiently long signal.

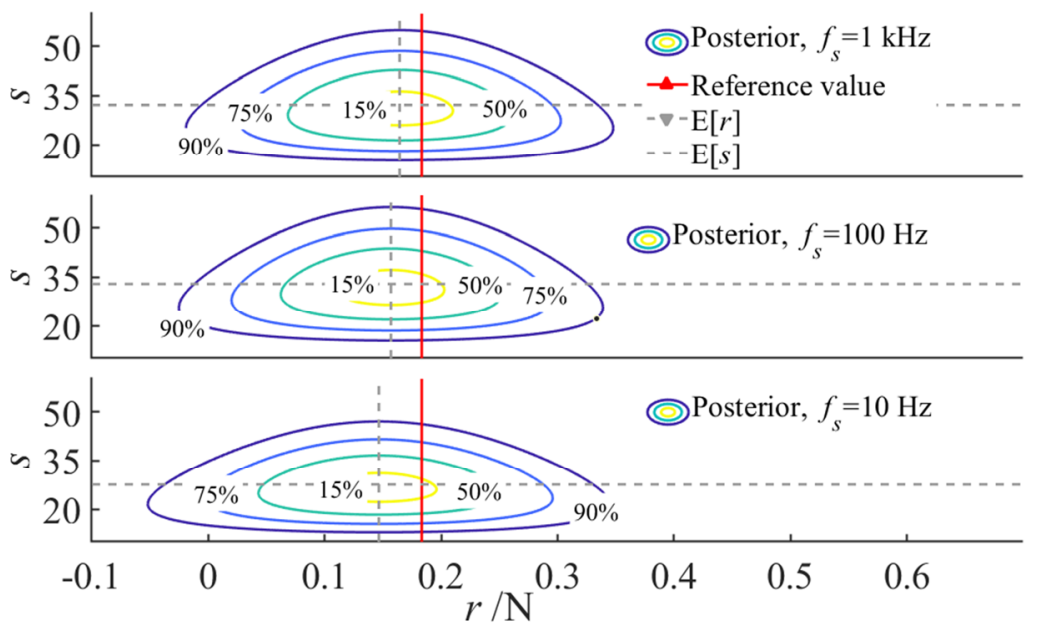

Fig. 14 The Coulomb friction force estimated for the reduced data set and different sampling rates

Since the natural frequency of the experimental setup is $2.9 \mathrm{~Hz}$, relatively high sampling rates of $1 \mathrm{kHz}$ and $100 \mathrm{~Hz}$ provided a reasonably good friction estimation regarding the diagnostics, which is further supported by the precision parameter. Nevertheless, when the sampling frequency is significantly reduced, in this case to $10 \mathrm{~Hz}$, the friction estimation is poor regardless of the fact that the Nyquist frequency criterion is satisfied. Thus, in order for the proposed analysis to achieve a reasonably good estimation, the sampling rate should be at least two orders of magnitude higher than the natural frequency.

\section{Conclusions}

In this paper, the friction force is chosen as a technical indicator for fast and efficient condition-based maintenance (CBM) due to the good correlation with degradation. The main goal was to propose a fast and efficient but technically simple friction estimation procedure that can be implemented in the condition-based maintenance strategy. In the free decay case, friction estimation can be performed by calculating the slope on two consecutive amplitudes. Since real-life situations regarding CBM involve small data sets, noise, and interference, the same approach could lead to a wrong estimation. Hence, the Bayesian analysis is used to estimate parameters and the related uncertainty. To avoid complex and time-consuming numerical methods, a conjugate prior model was used. In the numerical example, it appears that the gamma-exponential family of conjugate priors is not suitable for friction estimation while the normal-gamma family of conjugate priors can be efficiently used for that purpose. The proposed conjugate prior is two dimensional, which means that it estimates the friction force and the precision parameter. The latter was used as a control parameter, further supporting the estimated value acceptance when the value of interest cannot be directly measured. The proposed procedure was evaluated experimentally on the acquired acceleration free decay responses. An excellent correlation between the estimated and the recorded friction force was established. Furthermore, the precision parameter suggested a proper parameter estimation. The same analysis was performed on a reduced data set; a satisfactory friction estimation was obtained, with a small deviation compared to the full data set. In addition, the precision parameter suggested a valid estimation. 
In addition, the proposed analysis was tested regarding the data quality, i.e. the sampling rate sensitivity. For the analysed testbed, it has been shown that the diagnostic signal has to be at least two orders of magnitude higher than its natural frequency in order to obtain a relatively good estimation. The paper proposes an efficient friction force estimation procedure tested on a laboratory model that can be easily applied in less controlled environmental conditions.

\section{REFERENCES}

[1] J.Z. Sikorska, M. Hodkiewicz, L. Ma, Prognostic modelling options for remaining useful life estimation by industry, Mech. Syst. Signal Process. 25 (2011) 1803-1836. doi:10.1016/j.ymssp.2010.11.018.

[2] W. Caesarendra, T. Tjahjowidodo, B. Kosasih, A.K. Tieu, Integrated Condition Monitoring and Prognosis Method for Incipient Defect Detection and Remaining Life Prediction of Low Speed Slew Bearings, Machines. 5 (2017) 11. doi:10.3390/machines5020011.

[3] E. Quatrini, F. Costantino, G. Di Gravio, R. Patriarca, Condition-Based Maintenance-An Extensive Literature Review, Machines. 8 (2020) 31. doi:10.3390/machines8020031.

[4] W. Wang, A model to predict the residual life of rolling element bearings given monitored condition information to date, IMA J. Manag. Math. 13 (2002) 3-16. doi:10.1093/imaman/13.1.3.

[5] J. Salgueiro, G. Peršin, J. Vižintin, M. Ivanovič, B. Dolenc, On-line Oil Monitoring and Diagnosis, Strojniški Vestn. - J. Mech. Eng. 10 (2013) 604-612. doi:10.5545/sv-jme.2013.973.

[6] E. Pennestrì, V. Rossi, P. Salvini, P.P. Valentini, Review and comparison of dry friction force models, Nonlinear Dyn. 83 (2016) 1785-1801. doi:10.1007/s11071-015-2485-3.

[7] F.A.-B. V. Lampaert, J. Swevers, Experimental comparison of different friction model for accurate lowvelocity cracking, in: Proc. 10th Mediterr. Conf. Control Autom., Lisbon, 2002.

[8] D.A.H. Hanaor, Y. Gan, I. Einav, Static friction at fractal interfaces, Tribol. Int. 93 (2016) 229-238. doi:10.1016/j.triboint.2015.09.016.

[9] K. Nakano, Two dimensionless parameters controlling the occurrence of stick-slip motion in a 1-DOF system with Coulomb friction, Tribol. Lett. 24 (2006) 91-98. doi:10.1007/s11249-006-9107-7.

[10] M. De Simone, D. Guida, Modal Coupling in Presence of Dry Friction, Machines. 6 (2018) 8. doi:10.3390/machines6010008.

[11] R. Hoffmann, R. Liebich, Experimental and numerical analysis of the dynamic behaviour of a foil bearing structure affected by metal shims, Tribol. Int. 115 (2017) 378-388. doi:10.1016/j.triboint.2017.04.040.

[12] I. Hutchings, P. Shipway, Applications and case studies, in: Tribology, Elsevier, 2017: pp. 303-352. doi:10.1016/B978-0-08-100910-9.00009-X.

[13] G.W. Stachowiak, A.W. Batchelor, Engineering tribology, Second Edi, Butterworth-Heinemann, 2000.

[14] R. Tandler, N. Bohn, U. Gabbert, E. Woschke, Experimental investigations of the internal friction in automotive bush chain drive systems, Tribol. Int. 140 (2019) 105871. doi:10.1016/j.triboint.2019.105871.

[15] B.F. Liang, J.W., Feeny, Identifying Coulomb and Viscous Friction from Free-Vibration Decrements, Nonlinear Dyn. (1998) 337-347. https://doi.org/10.1023/A:1008213814102.

[16] J.-W. Liang, Identifying Coulomb and viscous damping from free-vibration acceleration decrements, J. Sound Vib. 282 (2005) 1208-1220. doi:10.1016/j.jsv.2004.04.034.

[17] W.T. Thomson, Theory of Vibration with Applications, Fourth, Springer US, Boston, MA, 1993. doi:10.1007/978-1-4899-6872-2.

[18] D. Kozelj, Z. Kapelan, G. Novak, F. Steinman, Investigating Prior Parameter Distributions in the Inverse Modelling of Water Distribution Hydraulic Models, Strojniški Vestn. - J. Mech. Eng. 60 (2014) 725-734. doi:10.5545/sv-jme.2014.1741.

[19] T. Ando, Bayesian Model Selection and Statistical Modeling, Taylor and Francis Group, New York, 2010.

[20] K. Worden, J.J. Hensman, Parameter estimation and model selection for a class of hysteretic systems using Bayesian inference, Mech. Syst. Signal Process. 32 (2012) 153-169. doi:10.1016/j.ymssp.2012.03.019.

[21] C. Andrieu, N. De Freitas, A. Doucet, I.M. Jordan, An introduction to MCMC for machine learning, Mach. Learn. 50 (2003) 5-43. doi:10.1023/A:1020281327116.

[22] B.P. Carlin;, T.A. Louis, Bayesian Methods for Data Analysis Third Edition, 2009. 
[23] J. Barle, D. Ban, M. Ladan, Maritime component reliability assessment and maintenance using Bayesian framework and generic data, in: Adv. Sh. Des. Pollut. Prev., 2010: pp. 181-188.

[24] M.J. Bernardo, A.F.M. Smith, Bayesian Theory, 3th ed., John Wiley \& Sons, 2000.

[25] Q. Gao, W. Chen, L. Lu, D. Huo, K. Cheng, Aerostatic bearings design and analysis with the application to precision engineering: State-of-the-art and future perspectives, Tribol. Int. 135 (2019) 1-17. doi:10.1016/j.triboint.2019.02.020.

[26] L. Marino, A. Cicirello, Experimental investigation of a single-degree-of-freedom system with Coulomb friction, Nonlinear Dyn. 99 (2020) 1781-1799. doi:10.1007/s11071-019-05443-2.

[27] M.E. Paté-Cornell, Uncertainties in risk analysis: Six levels of treatment, Reliab. Eng. Syst. Saf. 54 (1996) 95-111. doi:10.1016/S0951-8320(96)00067-1.

[28] R. Jordan-Cizelj, I. Vrbanić, Modeling Uncertainties when Estimating Component Reliability, Strojniški Vestn. - J. Mech. Eng. 49 (2003) 413-425.

[29] P.L. Green, Bayesian system identification of a nonlinear dynamical system using a novel variant of Simulated Annealing, Mech. Syst. Signal Process. 52-53 (2015) 133-146. doi:10.1016/j.ymssp.2014.07.010.

[30] S. Brooks, A. Gelman, G.L. Jones, X.-L. Meng, Handbook of Markov Chain Monte Carlo, CRC Press, 2011. https://doi.org/10.1201/b10905.

$\begin{array}{ll}\text { Submitted: } & 14.01 .2021 \\ \text { Accepted: } & 22.02 .2021\end{array}$
Stipe Perišić

Jani Barle

FESB, University of Split, R. Boškovića 32, 21000 Split, Croatia

Predrag Đukić

University department of professional studies, University of Split, Kopilica 5, 21000 Split, Croatia

Hinko Wolf

Faculty of Mechanical Engineering and Naval Architecture, University of Zagreb, Ivana Lučića 5, 10000 Zagreb, Croatia 